\title{
Use of Intraparticle Mass Transfer Parameters as a Design Tool for Catalyst Pellets
}

\author{
L. PETROV ${ }^{1,{ }^{*}}$, M. DAOUS ${ }^{1}$, Y. ALHAMED $^{1}$, A. AL-ZAHRANI ${ }^{1}$, Kh. MAXIMOV $^{2}$ \\ ${ }^{1}$ SABIC Chair in Catalysis, Chemical and Materials Engineering Department, Faculty of Engineering, King Abdulaziz University, PO Box \\ 80204, Jeddah 21589, Kingdom of Saudi Arabia \\ ${ }^{2}$ Institute of Catalysis, Bulgarian Academy of Sciences, Sofia 1113, Bulgaria
}

\begin{abstract}
A chromatographic method and a dynamic Wicke-Kallenbach method (DMWK) were used to determine the diffusion characteristics of two industrial copper containing catalysts. The first catalyst was used in nitrobenzene hydrogenation to aniline and the second was used in a low temperature water-gas shift reaction. Experimental results show that application of these two methods leads to similar results. Experimental data obtained allow for monitoring changes in the texture of the catalyst grains and intraparticle diffusivity of gaseous reagents at different states of the catalyst activity and use, which can be used as criteria for designing optimal industrial catalyst pellets.
\end{abstract}

Key words: effective diffusion coefficient; tortuosity; copper catalyst; pellet design

CLC number: O643 Document code: A

Received 2 February 2012. Accepted 2 May 2012.

*Corresponding author.Tel: +966-507589382; Fax: +966-2-6952257; E-mail: lpetrov@kau.edu.sa

This work was supported by SABIC Chair in Catalysis, Chemical and Materials Engineering Department, Faculty of Engineering, King Abdulaziz University, Kingdom of Saudi Arabia.

English edition available online at Elsevier ScienceDirect (http://www.sciencedirect.com/science/journal/18722067).

Catalyst shape and grain structure strongly influence their inter-particle and intra-particle heat and mass transfer characteristics and hence their performance in any industrial scale application. Thus, a proper design of a catalyst for industrial scale applications requires not only a proper attention to its catalytic characteristics but also a due attention to its shape and grain structure that allow for a better achievement of the desired heat and mass transfer in the catalyst grains and hence the required catalytic performance in the industrial reactors. Therefore, a purposeful design of industrial catalysts requires not only experience in the area of catalysis but also an in-depth knowledge of the process thermodynamic, kinetic and mechanism of the reaction and mass and heat transfer processes [1].

Inter- and intra-particle mass and heat transfer processes substantially affect the catalytic performance of a heterogeneous catalyst and the proceeding of an industrial scale catalytic reaction [2]. Therefore, optimal performance of a particular heterogeneous catalyst and successful executions of an industrial scale catalytic reaction demand proper attention to the design and formulation of the catalyst structure and its grain formation that allow for optimal inter- and intra-particle transfer processes.

The relevance and influence of mass and heat transfer phenomena on heterogeneous catalytic reaction processes are well established and presented in details in different references [3-6]. Among the important parameters of key importance in all theoretical treatments of catalytic processes are basic quantities related to the mass transfer in the catalyst grain, such as the porosity $\alpha$, effective diffusion

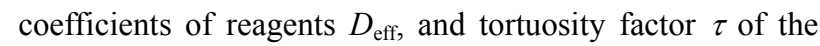
catalyst pores, or simply the tortuosity. To account for the influence of porosity on a diffusion process, the diffusion coefficient must be measured along with the tortuosity. Moreover, critical reviews of experimental methods to measure the effective diffusion coefficients in porous media are also given by different researchers [7-10].

Measurements of effective diffusion coefficients and tortuosity factors of industrial catalysts are usually performed on as received samples prepared by the catalyst producers. However, most catalysts need to be activated by special procedures before their actual use. The transformation of a catalyst into its active form is a complex process that results into changes in the chemical and physical properties of the catalysts accompanied by changes in the textural and mechanical properties of the catalyst grains.

Under operating conditions, industrial catalysts undergo further considerable changes in their chemical composition, crystal morphology, catalyst's texture, etc. At the same time, catalysts are steadily deactivated by one or more mean and mechanism including coking, sintering, fouling, phase transformation, and others. Such changes strongly affect the 
catalyst performance as well as the mass and heat transfer processes within the catalyst pellets and in the catalyst bed.

Only limited data are available in the literature on measurements of diffusion parameters such as the effective diffusion coefficient and tortuosity factor of catalyst pores performed on as received catalysts in their oxide state and after being activated in situ by a reduction step, as well as after being used and practically deactivated. Such data can be particularly useful for an optimum design of catalyst pellets.

Thus, the present work is concerned with the effect of the process of catalyst reduction and deactivation on the diffusion characteristics of industrial copper containing catalysts. Two catalyst samples used in two different types of catalytic processes, namely, nitrobenzene hydrogenation to aniline as a process representing typical catalytic organic synthesis, and a catalyst used in low temperature water-gas shift reaction (WGSR) as a typical catalytic process in the ammonia synthesis industry.

\section{Experimental}

\subsection{Catalysts}

\subsubsection{Copper-based catalyst}

The first tested catalyst is a copper-based catalyst used for the hydrogenation of organic compounds, such as nitrobenzene hydrogenation to aniline. It contains $50 \mathrm{wt} \% \mathrm{Cu}$ supported on activated kieselguhr and promoted with 1.5 $\mathrm{wt} \% \mathrm{Cr}_{2} \mathrm{O}_{3}$ and $1.0 \mathrm{wt} \% \mathrm{Ni}$. Commercial cylindrical pellets of $5 \times 5 \mathrm{~mm}$ particle size, $1.25 \mathrm{~kg} / \mathrm{L}$ bulk density, and 100 $\mathrm{kg} / \mathrm{cm}^{2}$ compression strength were used. The BET area of the catalyst is $119 \mathrm{~m}^{2} / \mathrm{g}$. Samples of various state of the catalyst as outlined below were subjected to diffusion characteristic measurements.

(a) Random samples (200 g) taken from various industrial containers of the oxide form of freshly prepared catalyst.

(b) Reduced samples of freshly prepared oxide catalyst. Samples were reduced in situ in the diffusion cell or the chromatographic column wherever the diffusion measurements were made. Such samples would emulate industrial copper oxide catalysts reduced in situ in the industrial reactor before use.

(c) Random samples of used and practically spent catalyst collected from the discharge of an industrial reactor.

Prior to diffusion measurements, the copper catalysts were first dried in the diffusion cells using argon flow at $393 \mathrm{~K}$ for $6 \mathrm{~h}$. For the case of in situ reduced samples, a flow of hydrogen at a gradually raised temperature to $473 \mathrm{~K}$ was employed for $3 \mathrm{~h}$ after the drying step with argon flow.

\subsubsection{Commercial copper-zinc-aluminum oxide catalyst}

The second tested catalyst is a commercial copper-zinc-aluminum oxide catalyst used in low temperature WGSR. The catalyst is composed of $38.0 \mathrm{wt} \% \mathrm{CuO}, 27.0$ $\mathrm{wt} \% \mathrm{ZnO}, 33.0 \mathrm{wt} \% \mathrm{Al}_{2} \mathrm{O}_{3}$, and $2.0 \mathrm{wt} \% \mathrm{Cr}_{2} \mathrm{O}_{3}$. It consists of $6 \times 6 \mathrm{~mm}$ cylindrical pellets of $1.25 \mathrm{~kg} / \mathrm{L}$ bulk density and $89.1 \mathrm{~m}^{2} / \mathrm{g}$ BET area.

In an industrial application, copper oxide as part of the catalyst composition is reduced in the industrial reactor under a stream of a mixture of hydrogen, carbon monoxide, and nitrogen at elevated temperatures. Thus, the diffusion characteristics in samples of the following different states of the catalyst were carried out.

(a) Random samples (200 g) taken from various industrial containers of the oxide form of freshly prepared catalyst.

(b) Reduced samples of freshly prepared oxide catalyst. Samples were reduced in situ in the diffusion cell or the chromatographic column wherever the diffusion measurements are made.

Prior to diffusion measurements, the WGSR catalyst samples were first dried in the diffusion cells using a flow of argon at $393 \mathrm{~K}$ for $6 \mathrm{~h}$. For the case of in situ reduced samples, hydrogen was gradually introduced to replace argon at the end of the drying step with a gradual increase in temperature to $473 \mathrm{~K}$. Sample reduction step was continued for $3 \mathrm{~h}$ after the drying step.

\subsection{Chemicals}

The following laboratory grade high purity gases were used: $99.999 \%$ argon dried over a 5A molecular sieve, electrolytic hydrogen purified of traces of oxygen using a Pd catalyst and dried over a 5A molecular sieve, and oxygen dried over a $5 \mathrm{~A}$ molecular sieve.

\subsection{Apparatus}

Diffusion measurements were carried out using two of the most popular and reliable experimental diffusion measurement methods, namely the chromatographic method (CM) and the dynamic method of Wicke-Kallenbach (DMWK).

Experimental measurements for both methods were carried out by using a HP 6890 gas chromatograph. Carrier and tracer gas employed in each experiment were first filtered, dried, and their pressure and flow rate regulated before being introduced into the diffusion measurement device (Chromatographic column in the case of CM and diffusion cell in case of DMWK). The diffusion measurements on all 
samples were carried out in the 293-433 K temperature range at atmospheric pressure.

For CM experiments, we used an $895 \mathrm{~mm}$ long and 11 $\mathrm{mm}$ internal diameter stainless steel column filled with the tested catalyst pellets and connected to the GC in place of normally used capillary columns into GC hot box. Gas pairs of $\mathrm{H}_{2}, \mathrm{He}$, and $\mathrm{Ar}$ were used as carrier and tracer gases in tests carried out on reduced catalyst samples. $\mathrm{Ar}$ and $\mathrm{O}_{2}$ were used as carrier and tracer in tests carried out on oxide catalyst samples. Tracer gas pulses $\left(1.0 \mathrm{~cm}^{3}\right)$ were dosed into the chromatographic column via a six-way sampling valve. The exit profile of each pulse was monitored by a thermal conductivity detector. The pressure difference between the entrance and the exit of the chromatographic column was monitored by an electronic differential manometer. In order to minimize dead volume, $0.8 \mathrm{~mm}$ internal diameter stainless steel tubes were used in all gas connections. Moreover, after each experimental run and before changing the used gas pair (tracer-carrier), the catalyst was purged in a flow of the next used carrier gas for $4 \mathrm{~h}$.

Diffusion measurements on a single pellet using the Wicke-Kallenbach method were carried out using a thermostatically controlled diffusion cell connected to the GC in place of the chromatographic column.

Figure 1 shows a schematic drawing of the diffusion cell. Special single catalyst pellet was prepared for these tests by

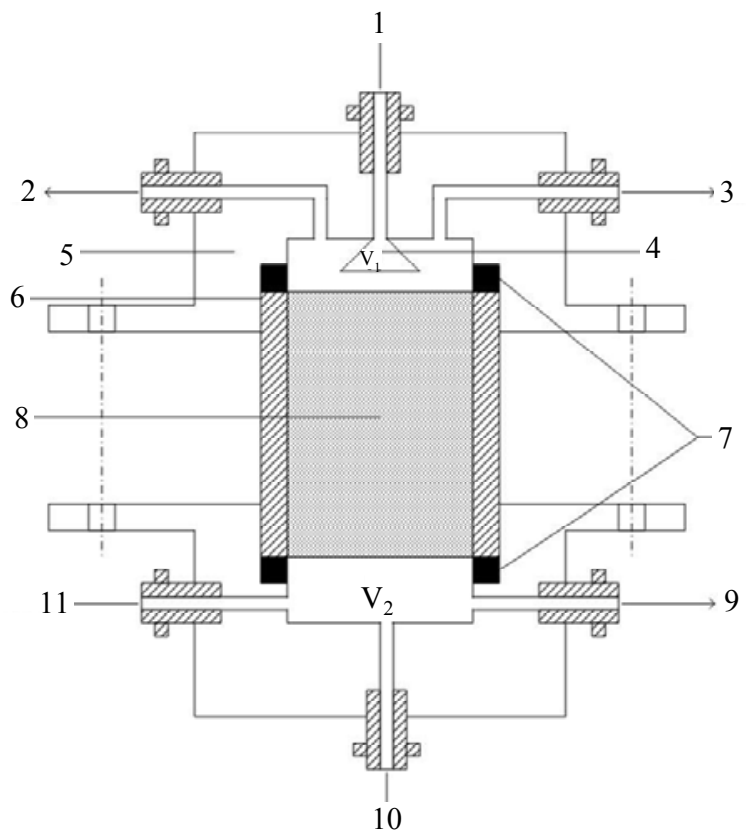

Fig. 1. Diffusion cell for dynamic method of Wicke-Kallenbach. 1 Tracing gas inlet; 2 Outlet from the upper volume; 3 Outlet to the differential manometer; 4 Device limiting the upper volume; 5 Cell's body; 6 Die; 7 Teflon O-rings; 8 Catalyst pellet; 9 Exit to the TCD; 10 Outlet to the differential manometer; 11 Carrier gas inlet to lower volume; $\mathrm{V}_{1}$ and $\mathrm{V}_{2}$ are upper and lower volumes, respectively. pelletizing $0.1 \mathrm{~mm}$ particles of crushed industrial catalyst pellets in a $10-\mathrm{mm}$ inside diameter and 17-mm high stainless steel die at regulated pressures similar to those used in the industrial pelletization of the studied catalysts. After that the two end flat surfaces of the formed pellet were then further polished and smoothed. The steel die containing the formed pressed catalyst was directly placed into the diffusion cell in such a way that insures a perpendicular trajectory of the diffusing gas component with respect to the pellet cross section.

Prior to conducting tests using the diffusion cell, two independent streams of carrier gas were passed through the upper and lower volumes of the diffusion cell separated by the catalyst pellet inside the die. A constant feed of 50 $\mathrm{cm}^{3} /$ min flow rate was passed through the upper volume, whereas a varied flow rate ranging between 20 and 200 $\mathrm{cm}^{3} /$ min was passed through the lower volume. Pairs of each of $\mathrm{H}_{2}, \mathrm{He}$, and $\mathrm{Ar}$ were used as carrier and tracer gases in reduced catalyst samples tests. $\mathrm{Ar}$ and $\mathrm{O}_{2}$ were used as carrier and tracer gases in tests carried out on catalyst samples in their oxide form.

Because it is particularly important to keep equal pressure in the upper and lower volumes of the diffusion cell, the pressure difference between these volumes was monitored by a precise electronic differential manometer within \pm 10 $\mathrm{Pa}$. The gas flow rate in the lower volume of the diffusion cell was controlled by Bronkhorst mass flow controller within $\pm 1 \%$ of the set point, while the temperatures of the thermostatically controlled chromatographic column and diffusion cell were kept within $\pm 0.1 \mathrm{~K}$. Moreover, after each experimental run and before changing the used gas pair (tracer-carrier), the catalyst was purged in a flow of the next used carrier gas for $4 \mathrm{~h}$.

\subsection{Data processing}

A specially designed computer program was developed to carry out the required mass transfer parameter calculations based on used models and data generated in the study.

\subsection{A brief background on used methods}

It is not well established which experimental method of measuring catalyst diffusion parameters gives the most reliable results. It is known, however, that only dynamic methods of determining diffusion parameters take into account the effect and contribution of catalyst dead pores on the mass transfer process within the catalyst. It is therefore expected that the dynamic methods of DMWK and CM, which are commonly used for such purposes, would give the most reliable and accurate data. 


\subsubsection{Chromatographic method}

Different variants of the chromatographic method for transport parameter determination were developed over the past years [11-14]. All of these methods, however, are based on the Kubin-Kucera model [15-17]. According to this model, the moments of chromatographic peak at the column outlet are correlated with the following mass transfer parameters: the axial dispersion coefficient, $E_{\mathrm{a}}$, the external mass transfer coefficient, $k_{f}^{\text {ext }}$, internal mass transfer coefficient, $k_{f}^{\text {int }}$, and the effective diffusion coefficient in the catalyst pores, $D_{\text {eff. }}$.

According to the Kubin-Kucera model, a linear dependence exists between the height of the theoretical plate (HETP) of the chromatographic column and the linear velocity of the carrier gas $u$. At high linear velocities of the carrier gas, we have

$$
H=A_{0}+B \cdot u
$$

Equation (1) describes a straight line in the $\mu_{1 \text { corr }}-1 / u$ coordinates, where $\mu_{1 \text { corr }}$ is the first absolute moment of the chromatographic peak corrected for dead volume of the system. The slope of this line gives the value of the coefficient $B$, whereas the intercept of the ordinate is equal to the coefficient $A_{0}$. From $B$ one can estimate the effective diffusion coefficient $D_{\text {eff }}$ for a porous catalyst, while from $A_{0}$ the axial dispersion coefficient $E_{\mathrm{a}}$ can be estimated.

To determine the tortuosity, we used the medium transport pore model (MTPM). This method is successfully applied to cylindrical commercial catalysts of pellet sizes within $75 \%-90 \%$ of column's internal diameter. According to this model, the effective diffusion coefficient, $D_{\text {eff }}$, at low concentrations of the tracer gas is given by

$$
\frac{1}{D_{\text {eff }}}=\frac{\tau}{\alpha \cdot D_{\mathrm{AB}}}+\frac{\tau}{r_{\mathrm{e}} \cdot \alpha \cdot D_{\mathrm{KA}}}
$$

where $D_{\mathrm{KA}}$ is the Knudsen's diffusion coefficient for cylindrical pores with an equivalent pore radius $r_{\mathrm{e}}, D_{\mathrm{AB}}$ is the molecular diffusion coefficient, $\alpha$ is the porosity of catalyst pellets, and $\tau$ is the tortuosity factor. Replacing the expressions for $D_{\mathrm{KA}}$ and $D_{\mathrm{AB}}$ given by Eqs. (3) and (4), respectively, into Eq. (2) yields Eq. (5), which allows for estimating catalyst pellets tortuosity factor $\tau$ and the equivalent pore radius $r_{\mathrm{e}}$ based on effective diffusion measurements in the catalyst pellets using different pairs of gases [18].

$$
\begin{gathered}
D_{\mathrm{KA}}=r_{\mathrm{e}}\left(\frac{32 R_{\mathrm{g}} T}{9 \pi M_{\mathrm{a}}}\right)^{0.5} \\
D_{\mathrm{AB}}=\frac{5.06 T^{1.75}}{\left(V_{\mathrm{A}}^{0.5}+V_{\mathrm{B}}^{1 / 3}\right)^{2} p^{1.286}}\left(\frac{1}{M_{\mathrm{A}}}+\frac{1}{M_{\mathrm{B}}}\right)^{0.5} \\
\frac{1}{D_{\mathrm{eff}} \cdot M_{\mathrm{A}}^{0.5}}=\frac{\tau}{\alpha \cdot r_{\mathrm{e}}}\left(\frac{9 \pi}{32 \cdot R \cdot T}\right)^{0.5}+\frac{\tau}{\alpha}\left(\frac{1}{D_{\mathrm{AB}} \cdot M_{\mathrm{A}}^{0.5}}\right)
\end{gathered}
$$

\subsubsection{Dynamic method of Wicke-Kallenbach}

The theory and applications of the dynamic method of Wicke-Kallenbach are well established [18-22]. More recently [23], the characteristics of the Wicke-Kallenbach and Graham's diffusion cells were studied. A Wicke-Kallenbach cell for effective diffusion and tortuosity factor measurements at pressures up to $10 \mathrm{~atm}$ and temperatures up to 600 ${ }^{\circ} \mathrm{C}$ is described in [24].

The equation for the first absolute moment in this theory is given by

$$
\mu_{1}=\frac{\alpha \cdot L^{2}\left(3 \frac{A}{L} D_{\text {eff }}+F\right)}{6 D_{\text {eff }}\left(\frac{A}{L} D_{\text {eff }}+F\right)}
$$

When the effective diffusion is determined by a dynamic method, it is necessary to determine accurately the dead volume of the system. If we assume that no axial dispersion occurs in the connecting gas lines and that a perfect mixing is achieved in the two volumes of the diffusion cell, the value of the experimentally observed first absolute moment is determined by the expression

$$
\mu_{1 \mathrm{corr}}=\mu_{1 \mathrm{obs}}-\frac{V_{1}}{F_{1}}-\frac{V_{2}}{F_{2}}-\frac{t_{0}}{2}
$$

where $t_{0} / 2$ is a correction for the impulse duration.

The effective diffusion coefficient $D_{\text {eff }}$ can be determined by combining equations (6) and (7).

$$
\mu_{1 \mathrm{corr}}=\frac{\alpha \cdot L^{2}}{6 D_{\mathrm{eff}}}+\frac{A \cdot D_{\mathrm{eff}} \cdot \mu_{1 \mathrm{corr}}}{L \cdot F}+\frac{\alpha \cdot A \cdot L}{F \cdot D_{\mathrm{eff}}}
$$

We used Nelder-Mead minimization algorithm [25] to calculate from equation (8) the best estimate of $D_{\text {eff. }}$ The criterion of the minimization was the minimum sum of square difference $S$ between $\mu_{1 \text { corr }}$ and $\mu_{1 \text { cal }}$ given by equation (9) below:

$$
S=\sum_{n}\left(\mu_{1 \mathrm{corr}}-\mu_{1 \mathrm{cal}}\right)^{2}
$$

where $n$ is the number of experimental measurements.

The tortuosity factor $\tau$ for pores with equivalent radius $r_{\mathrm{e}}$ was calculated by the following equation:

$$
\tau=\frac{\alpha D_{\mathrm{A}\left(r_{\mathrm{e}}\right)}}{D_{\text {eff }}}
$$

where $D_{\mathrm{A}(r)}$ is dependent on the ratio between the molecular and Knudsen diffusion and is defined as [22]

$$
\frac{1}{D_{\mathrm{A}\left(r_{\mathrm{e}}\right)}}=\frac{1}{D_{\mathrm{AB}}}+\frac{1}{D_{\mathrm{KA}\left(r_{\mathrm{e}}\right)}}
$$

\section{Results and discussion}

\subsection{Chromatographic method}


In all $\mathrm{CM}$ experimental measurements, three random samples from the same catalyst batch were tested at each experimental condition reported here. Five measurements were carried out on each sample and the average value of all measurements on the three samples at a given experimental condition is reported. It is worth mentioning that uncertainty on repeated measurements on the same sample from a given batch of a catalyst is $11.3 \%$, while that based on different samples from the same batch amounts to $20.1 \%$. The increase in the uncertainty is attributed to the existing nonhomogeneity among catalyst pellets in a given batch and probably due to the resulting changes in column filling from one experiment to the other when using different samples.

Values of $D_{\text {eff }}$ obtained by $\mathrm{CM}$ at different temperatures for the oxide and reduced forms of the copper and WGSR catalyst studied in this investigation are presented in Table 1. It should be noted, however, that these obtained values are valid for diffusion in pores with radius $r_{\mathrm{e}}$ because the Knudsen diffusion coefficient estimated here is for a pore radius equal to the mean radius $r_{\mathrm{e}}$ of the catalyst pores.

Table 1 The effective diffusion coefficients $D_{\text {eff }}$ for oxide and reduced forms of the $\mathrm{Cu}$ catalyst and WGSR catalyst estimated by $\mathrm{CM}$

\begin{tabular}{|c|c|c|c|}
\hline Catalyst & $T / \mathrm{K}$ & Gas pair & $D_{\text {eff }} \cdot 10^{2} /\left(\mathrm{cm}^{2} / \mathrm{s}\right)$ \\
\hline \multirow[t]{3}{*}{$\mathrm{Cu}_{\text {oxide }}$} & 293 & $\mathrm{O}_{2}-\mathrm{Ar}$ & 0.32 \\
\hline & 353 & $\mathrm{O}_{2}-\mathrm{Ar}$ & 0.39 \\
\hline & 433 & $\mathrm{O}_{2}-\mathrm{Ar}$ & 0.45 \\
\hline \multirow[t]{3}{*}{$\mathrm{Cu}_{\text {oxide }}$} & 293 & $\mathrm{Ar}-\mathrm{O}_{2}$ & 0.29 \\
\hline & 353 & $\mathrm{Ar}-\mathrm{O}_{2}$ & 0.39 \\
\hline & 433 & $\mathrm{Ar}-\mathrm{O}_{2}$ & 0.46 \\
\hline \multirow[t]{3}{*}{$\mathrm{Cu}_{\text {reduced }}$} & 293 & $\mathrm{He}-\mathrm{H}_{2}$ & 1.22 \\
\hline & 353 & $\mathrm{He}-\mathrm{H}_{2}$ & 1.42 \\
\hline & 433 & $\mathrm{He}-\mathrm{H}_{2}$ & 1.56 \\
\hline \multirow[t]{3}{*}{$\mathrm{Cu}_{\text {reduced }}$} & 293 & $\mathrm{H}_{2}-\mathrm{Ar}$ & 0.85 \\
\hline & 353 & $\mathrm{H}_{2}-\mathrm{Ar}$ & 2.44 \\
\hline & 433 & $\mathrm{H}_{2}-\mathrm{Ar}$ & 3.25 \\
\hline \multirow[t]{3}{*}{$\mathrm{Cu}_{\text {reduced }}$} & 293 & $\mathrm{Ar}-\mathrm{H}_{2}$ & 0.51 \\
\hline & 353 & $\mathrm{Ar}-\mathrm{H}_{2}$ & 1.62 \\
\hline & 433 & $\mathrm{Ar}-\mathrm{H}_{2}$ & 2.52 \\
\hline \multirow[t]{3}{*}{ WGSR $_{\text {oxide }}$} & 293 & $\mathrm{O}_{2}-\mathrm{Ar}$ & 0.39 \\
\hline & 353 & $\mathrm{O}_{2}-\mathrm{Ar}$ & 0.45 \\
\hline & 433 & $\mathrm{O}_{2}-\mathrm{Ar}$ & 0.51 \\
\hline \multirow[t]{3}{*}{ WGSR $_{\text {oxide }}$} & 293 & $\mathrm{Ar}-\mathrm{O}_{2}$ & 0.37 \\
\hline & 353 & $\mathrm{Ar}-\mathrm{O}_{2}$ & 0.41 \\
\hline & 433 & $\mathrm{Ar}-\mathrm{O}_{2}$ & 0.49 \\
\hline \multirow[t]{3}{*}{ WGSR $_{\text {reduced }}$} & 293 & $\mathrm{He}-\mathrm{H}_{2}$ & 1.52 \\
\hline & 353 & $\mathrm{He}-\mathrm{H}_{2}$ & 1.59 \\
\hline & 433 & $\mathrm{He}-\mathrm{H}_{2}$ & 1.76 \\
\hline \multirow[t]{3}{*}{ WGSR $_{\text {reduced }}$} & 293 & $\mathrm{H}_{2}-\mathrm{Ar}$ & 0.92 \\
\hline & 353 & $\mathrm{H}_{2}-\mathrm{Ar}$ & 2.66 \\
\hline & 433 & $\mathrm{H}_{2}-\mathrm{Ar}$ & 4.29 \\
\hline \multirow[t]{3}{*}{ WGSR $_{\text {reduced }}$} & 293 & $\mathrm{Ar}-\mathrm{H}_{2}$ & 0.71 \\
\hline & 353 & $\mathrm{Ar}-\mathrm{H}_{2}$ & 1.79 \\
\hline & 433 & $\mathrm{Ar}-\mathrm{H}_{2}$ & 2.63 \\
\hline
\end{tabular}

Based on the MTPM theory used, experimental measurements of the effective diffusion coefficient at a given temperature using different gas couples allow for determining the tortuosity factor $\tau$ and the equivalent pore radius $r_{\mathrm{e}}$ from the slope and the intercept of a linear plot of $1 / D_{\text {eff }}$ versus $1 / D_{\mathrm{AB}}$ form as indicated by Eq. (5). For this purpose, experimental runs with different gas pairs were carried out using $\mathrm{He}, \mathrm{O}_{2}$ or Ar as tracer gases and $\mathrm{H}_{2}, \mathrm{Ar}$ or $\mathrm{O}_{2}$ as carrier gases. From this data we were able to estimate the values of the pore tortuosity factor $\tau$ for all the gas couples at different temperatures. $r_{\mathrm{e}}$ values at different temperatures for the two studied catalysts in oxide and reduced forms are given in Table 2.

Table 2 The tortuosity factor $\tau$ and equivalent pore radius $r_{\mathrm{e}}$ obtained from the CM for oxide and reduced forms of the $\mathrm{Cu}$ and WGSR catalysts

\begin{tabular}{lcccc}
\hline Catalyst & $T / \mathrm{K}$ & $\tau_{\text {oxide }}$ & $\tau_{\text {reduced }}$ & $r_{\mathrm{e}} \cdot 10^{-7} /(\mathrm{cm})$ \\
\hline Cu catalyst & 293 & 8.5 & 5.4 & 2.3 \\
& 353 & 5.2 & 3.6 & 2.3 \\
WGSR catalyst & 433 & 3.9 & 2.9 & 2.4 \\
& 293 & 11.7 & 7.4 & 3.2 \\
& 353 & 8.6 & 5.1 & 3.2 \\
& 433 & 5.2 & 3.7 & 3.3 \\
\hline
\end{tabular}

\subsection{Dynamic method of Wicke-Kallenbach}

Similarly, in all DMWK experimental measurements, three random samples from the same catalyst batch were tested at each experimental condition reported here. Five measurements were carried out on each sample and the average value of these measurements on all three samples is reported. However, the uncertainty on repeated measurements on the same sample from a given batch of a catalyst using DMWK is only $7.8 \%$, while that based on different samples from the same batch amounts to $14.4 \%$. This increase in the uncertainty is attributed to the existing nonhomogeneity among catalyst pellets in a given batch.

The lower uncertainty in experimental measurement based on DMWK than that based on CM is attributed to differences in sample preparation in the two methods, the absence of axial dispersion and the much smaller influence of reversible adsorption of the tracer gas in DMWK.

The effective diffusion coefficients $D_{\text {eff }}$ and tortuosity factors $\tau$ for the two studied catalysts in their oxide and reduced forms estimated by DMWK at different temperatures are presented in Table 3.

Values of $D_{\text {eff }}$ found in this study and presented in Tables 1 and 3 are consistent with those reported by previous studies on supported copper catalyst for nitrobenzene hydrogenation [26,27]. These previous studies, however, were conducted on the oxide form of the catalyst only. 
Table 3 Effective diffusion coefficients $D_{\text {eff }}$ and tortuosity factors $\tau$ for oxide and reduced forms of the $\mathrm{Cu}$ and WGSR catalysts estimated by DMWK

\begin{tabular}{lcccc}
\hline Catalyst & $T / \mathrm{K}$ & $\mathrm{Gas}$ pair & $D_{\text {eff }} 10^{2} /\left(\mathrm{cm}^{2} / \mathrm{s}\right)$ & $\tau$ \\
\hline $\mathrm{Cu}_{\text {oxide }}$ & 293 & $\mathrm{O}_{2}-\mathrm{Ar}$ & 0.36 & 9.3 \\
& 353 & $\mathrm{O}_{2}-\mathrm{Ar}$ & 0.41 & 6.0 \\
$\mathrm{Cu}_{\text {reduced }}$ & 433 & $\mathrm{O}_{2}-\mathrm{Ar}$ & 0.49 & 5.2 \\
& 293 & $\mathrm{H}_{2}-\mathrm{Ar}$ & 1.85 & 7.0 \\
& 353 & $\mathrm{H}_{2}-\mathrm{Ar}$ & 2.91 & 5.2 \\
$\mathrm{Cu}_{\text {reduced }}$ & 433 & $\mathrm{H}_{2}-\mathrm{Ar}$ & 3.72 & 4.7 \\
& 293 & $\mathrm{Ar}-\mathrm{H}_{2}$ & 1.25 & 3.7 \\
& 353 & $\mathrm{Ar}-\mathrm{H}_{2}$ & 2.16 & 2.0 \\
WGSR $_{\text {oxide }}$ & 433 & $\mathrm{Ar}-\mathrm{H}_{2}$ & 3.12 & 1.6 \\
& 293 & $\mathrm{O}_{2}-\mathrm{Ar}$ & 0.42 & 12.3 \\
& 353 & $\mathrm{O}_{2}-\mathrm{Ar}$ & 0.48 & 8.1 \\
& 433 & $\mathrm{O}_{2}-\mathrm{Ar}$ & 0.55 & 5.4 \\
WGSR $_{\text {reduced }}$ & 293 & $\mathrm{H}_{2}-\mathrm{Ar}$ & 1.41 & 9.8 \\
& 353 & $\mathrm{H}_{2}-\mathrm{Ar}$ & 3.13 & 6.8 \\
& 433 & $\mathrm{H}_{2}-\mathrm{Ar}$ & 4.71 & 4.5 \\
& 293 & $\mathrm{Ar}-\mathrm{H}_{2}$ & 1.13 & 5.3 \\
WGSR $_{\text {reduced }}$ & 353 & $\mathrm{Ar}-\mathrm{H}_{2}$ & 2.23 & 3.2 \\
& 433 & $\mathrm{Ar}-\mathrm{H}_{2}$ & 3.20 & 2.5 \\
\hline & & & &
\end{tabular}

\subsection{Diffusion parameters of deactivated Cu catalysts}

Table 4 presents results of measurements of the diffusion parameters investigated at different states and different temperatures of the $\mathrm{Cu}$ catalyst. These include the as-received fresh oxide catalyst, the fresh reduced catalyst, and two different states of deactivated catalyst after 1000 and $3000 \mathrm{~h}$ of on-stream use.

\subsection{Dependence of the tortuosity factor on pelletizing} pressure

Table 4 Effect of catalyst reduction and deactivation on $D_{\text {eff }} \tau$, and $r_{\mathrm{e}}$ of the copper catalyst measured by both CM and DMWK

\begin{tabular}{lcccccccc}
\hline \multirow{2}{*}{ Catalyst } & & \multicolumn{3}{c}{$\mathrm{CM}$} & & \multicolumn{2}{c}{ DMWK } \\
\cline { 3 - 5 } \cline { 8 - 9 } & & $\begin{array}{c}D_{\text {eff }} / \\
\left(\mathrm{cm}^{2} / \mathrm{s}\right)\end{array}$ & $\tau$ & $\begin{array}{c}r_{\mathrm{e}} \cdot 10^{-7} \\
(\mathrm{~cm})\end{array}$ & & $\begin{array}{c}D_{\text {eff }} / \\
\left(\mathrm{cm}^{2} / \mathrm{s}\right)\end{array}$ & $\tau$ \\
\hline $\mathrm{Cu}_{\text {fresh oxide }}$ & 293 & 0.32 & 7.0 & 1.9 & & 0.36 & 8.2 \\
& 353 & 0.39 & 5.2 & 2.0 & & 0.41 & 5.6 \\
$\mathrm{Cu}_{\text {in situ reduced }}$ & 433 & 0.46 & 3.9 & 2.0 & & 0.49 & 5.0 \\
& 293 & 0.68 & 5.9 & 2.3 & & 1.55 & 3.4 \\
& 353 & 2.03 & 4.4 & 2.3 & & 2.54 & 1.7 \\
$\mathrm{Cu}_{\text {affer } 1000 \text { h on stream }}$ & 293 & 0.54 & 4.1 & 1.7 & & 0.76 & 3.0 \\
& 353 & 1.63 & 3.1 & 1.8 & & 1.89 & 1.4 \\
& 433 & 2.31 & 2.3 & 1.8 & & 2.55 & 1.2 \\
$\mathrm{Cu}_{\text {affer 3000 h on stream }}$ & 293 & 0.32 & 2.8 & 1.3 & & 0.38 & 2.5 \\
& 353 & 0.96 & 2.1 & 1.3 & & 1.08 & 1.0 \\
& 433 & 1.36 & 1.6 & 1.4 & & 1.55 & 0.8 \\
\hline
\end{tabular}

Tortuosity of a catalyst pellet can be manipulated and controlled by changing the condition of the pelletizing process. Knowledge of the value of the tortuosity factor $\tau$ provides a tool for estimating the effective diffusion coefficient $D_{\text {eff }}$ for an arbitrary gas couple. This is because $\tau$ is independent of the properties of diffusing gases and is only related and influenced by the texture of the porous solid.

Table 5 presents results of experimental data on the influence of pelletizing pressure on important physical parameters including porosity, surface area, mean pore radius, and tortuosity of the $\mathrm{Cu}$ and WGSR catalysts.

Table 5 Dependence of the copper and WGSR catalyst pellet properties on pelletizing pressure

\begin{tabular}{lccccc}
\hline \multirow{2}{*}{$\begin{array}{l}\text { Catalyst } \\
\end{array}$} & $\begin{array}{c}\text { Pelletized } \\
\text { pressure }\left(\mathrm{kg} / \mathrm{cm}^{2}\right)\end{array}$ & $\begin{array}{c}\text { Porosity } \\
(\%)\end{array}$ & $\begin{array}{c}\text { Prevailing } \\
\text { pore size }(\mathrm{nm})\end{array}$ & $\begin{array}{c}\text { Surface area } \\
\left(\mathrm{m}^{2} / \mathrm{g}\right)\end{array}$ & $\tau$ \\
\hline $\mathrm{Cu} \quad$ before pelletizing & 47 & 1500 & 119.0 & - \\
& 1000 & 32 & 830 & 119.0 & 8.6 \\
& 1500 & 23 & 490 & 119.0 & 7.0 \\
& 3000 & 14 & 270 & 119.0 & 6.1 \\
\multirow{4}{*}{ WGSR } & 2000 & 41 & $15-30$ & 89.0 & 10.1 \\
& 2500 & 37 & $15-27$ & 89.0 & 9.7 \\
& 3000 & 33 & $15-25$ & 89.0 & 9.3 \\
& 3500 & 29 & $15-23$ & 89.0 & 8.7 \\
& 4000 & 29 & $14-21$ & 88.0 & 8.1 \\
& 5000 & 29 & $14-20$ & 87.0 & 7.5 \\
\hline
\end{tabular}

\subsection{Comparison of the methods for diffusion characteristic measurements}

The experimental work performed using CM and DMWK for estimation of transport parameters and obtained results confirm that both selected methods are equivalent and low time consuming, which made them attractive for use in industrial laboratories.

Compared to the CM, the DMWK has some advantages. The most important one is the opportunity to avoid the influence of side effects, such as an axial dispersion and external diffusion, on the value of the $D_{\text {eff. }}$ By using DMWK, it is easier to consider the influence of the pelletizing conditions and pellet size on the diffusion characteristics of catalyst. In some cases, the advantage is the fact that this method needs a very small amount of catalyst; even with only several pellets is possible to perform the experimental measurements. However, for the industrial catalysts always exist some non-homogeneity in the composition and structure from pellet to pellet, using a small amount of pellets is not recommendable. The more reliable results could be obtained if the DMWK cell contains many pellets and several successful measurements with different catalyst samples are done. From this point of view, the CM is preferable. In any case, the best results should be obtained when both methods 
are used.

The existence of external diffusion through voids of the catalyst bed in the column and axial dispersion taking place in the chromatographic methods has considerable influence on experiments under such measurements, which tends to mask the differences between structures of different samples. This is the reason that although two examined catalysts are manufactured by using different technologies and have different chemical composition, no substantial differences in their diffusion characteristics due to reduction were observed as evident by data of Tables 1 and 2. However, existing differences between the two states of the catalyst sample (oxide and reduced) are more evident by the results of the DMWK, which does not suffer from interference of diffusion through bed voids or axial dispersion.

Differences in diffusion characteristics between the two used methods lead to small to moderate differences between their measurement results. Depending on gas pairs used and temperature employed, discrepancies between measured values of $D_{\text {eff }}$ by the two methods range from $8.6 \%$ to $40 \%$. Comparison of data presented in Tables 1 and 2 presenting CM measurements with those in Table 3 of DMWK for the two catalysts at similar measurement conditions reveals that differences between the two methods diminish at higher temperature probably as a result of the decrease in catalyst pellet tortuosity, which diminishes the masking effects of void diffusion and axial dispersion taking place in the chromatographic column. It is worth noting that in both methods values of the $D_{\text {eff }}$ depend to some extent on the gas pairs used in the diffusion measurements as a result of the differences in physical properties of the gas pairs used.

\subsection{Factors affecting the diffusion parameters}

Based on the concept of tortuosity $\tau$ of a catalyst pellet as a measure of the ratio of the distance a diffusing species must travel between two points far apart in the pellet and the geometric distance between these two points, early theoretical predictions of this factor gave values of $\tau=1.44$ [3].

The differences in the values of the tortuosity factor $\tau$ estimated by two experimental methods for the oxide state of both catalysts and reported in Tables 2 and 3 arise from differences in the theoretical and experimental approaches employed in establishing this factor by these two methods. Thus, a direct comparison between the values obtained by the two methods is inappropriate because values of the tortuosity factor $\tau$ obtained by $\mathrm{CM}$ and reported in Table 2 were estimated by using all experimental data generated on the catalyst based on all combination of gas pairs used at each temperature. This provides a better smoothed value for tortuosity than that established by DMWK in which $\tau$ values are based only on single point measurements.
Values of tortuosity factor found in this study range from 2.5 to 12.3 for WGSR catalyst and 1.6 to 8.5 for $\mathrm{Cu}$ catalyst. This compares well with data reported in the literature for similar industrial catalysts. Values of tortuosity factor reported by Satterfield predominantly range between 2 and 4 [2]. Wang and Smith [28] reported average value of $\tau$ for industrial catalysts around 8 when both Knudsen and molecular diffusions were taken into account. For many industrial catalysts, such as high temperature WGSR, values for $\tau$ as high as 11.1 were reported and as high as 12.5 for ammonia synthesis catalyst [29]. Scott and Dullien [30] reported $\tau$ values of 4.53 for diatomaceous earth, while Diesler and Wilhelm [31] reported values of 5-10 for spherical activated alumina. More recently Metaxas and Papayanakos [32] reported a value of 3.75 for $\mathrm{Ni} / \mathrm{Al}_{2} \mathrm{O}_{3}$. Tortuosity factors reported in the literature for industrial catalysts portray values much higher than those predicted theoretically. No upper limit on the values of $\tau$ of an industrial catalyst is known.

The tortuosity factor is independent of the properties of the diffusing gases and is only affected by the resulting texture of the porous solid. However, its value is strongly dependent on the catalyst pellet composition and the method of preparation and pelletization. Moreover, catalyst pellet tortuosity is also affected by its pretreatment and activation process as well as by the conditions of the process in which it is employed.

\subsubsection{Catalyst preparation}

The two catalysts used in this study were prepared by different methods, have different chemical compositions, and are used in different industrial processes. The $\mathrm{Cu}$ catalyst is prepared by $\mathrm{Cu}$ precipitation on the carrier (activated kieselguhr), while WGSR catalyst is prepared by co-precipitating $\mathrm{Cu}, \mathrm{Zn}$, and $\mathrm{Al}$ oxides. The resulting transport properties of the $\mathrm{Cu}$ catalyst are strongly influenced by the corresponding carrier properties and not much by its preparation conditions. The transport properties of the WGSR catalyst, on the other hand, are formed during its preparation and is very much influenced and modified by its preparation conditions.

Data of Table 5 clearly show that tortuosity factor strongly depends on the pelletizing pressure. Table 5 further shows that up to a certain upper pressure value, the tortuosity as well as the average pore size decreases with increasing the pelletizing pressure. A decrease in the tortuosity resulting from higher pelletizing pressure might compensate for the accompanied decrease in the prevailing average pore size to maintain an unchanged mass transfer rate within the pores of the catalyst. It is also expected that up to certain pressure values the catalyst activity as a consequence of the 
above argument would not be strongly affected by changing the pelletization pressure. It should be recognized, however, that the pelletizing pressure is not the only factor in the catalyst pellet preparation process that affects its final property and performance. Other factors that are known to influence catalyst properties and performance include many other factors, such as the micro-structure of the material, chemical properties, rheological properties of the material prior to pelletization, water content, presence of additives that decrease the friction between the pellet and walls of the die of the tablet press. Therefore, it is envisaged that the utilization of the tortuosity factor and the effective diffusion coefficient are useful tools for selecting the optimum pelletizing conditions of any catalytic system.

\subsubsection{Catalyst reduction}

Freshly prepared industrial hydrogenation $\mathrm{Cu}$ catalysts are first reduced in the industrial reactor under stream of hydrogen and nitrogen mixture at temperatures not exceeding $250{ }^{\circ} \mathrm{C}$. WGSR Cu-Zn-Al catalyst is reduced either by a mixture of hydrogen and nitrogen or by a mixture of $\mathrm{H}_{2}$ and $\mathrm{CO}$ at temperatures between 250 and $300{ }^{\circ} \mathrm{C}$. The process of catalyst reduction is a crucial step in forming a highly active catalyst. It is obvious that the reduction mechanism of the two catalysts will be different and proceed in different manner. During this process, which is affected by many factors, the active phases of the catalysts are formed and changes in the catalyst texture and mechanical properties take place. These changes might have substantial effect on the mass transfer processes in the catalyst grains and in the catalyst bed. Most chemical and physical processes related to catalyst reduction are well studied, but limited attention has been given to study the changes in the transport properties of catalysts resulting from catalyst reduction process. The change in pore structure of alumina after hydrogen treatment was reported by Kirszensztejn and Bell [33].

Data in Tables 1-3 show that the process of catalyst reduction results in a decrease in the tortuosity factor. Experimental data in Tables 2 and 3 show that observed $\tau$ values of the reduced form of both catalysts are smaller when a tracer gas of higher molecular weight is used. As shown in Tables $1-3$, a substantial increase in $D_{\text {eff }}$ is observed for both catalysts upon their reduction. The observed changes in $D_{\text {eff }}$ values are temperature dependent. The higher the temperature, the larger the difference between $D_{\text {eff }}$ of the oxide and reduced forms of the catalyst. The difference in $D_{\text {eff }}$ measured by $\mathrm{CM}$ varies from $277 \%$ at $293 \mathrm{~K}$ to $576 \%$ at $533 \mathrm{~K}$ for both catalysts. On the other hand, changes in $D_{\text {eff }}$ values between the oxide and reduced form of the catalyst observed by using DMWK are different for the catalyst, but in both cases are larger than those observed by CM meas- urements. In both catalyst states investigated, values of $D_{\text {eff }}$ of WGSR catalyst are larger than those of $\mathrm{Cu}$ catalyst.

The effect of catalyst activation by reduction on catalyst transport properties should obviously be taken into account in reactor modeling and in establishing the optimum operating conditions in the industry [34-36].

\subsubsection{Catalyst deactivation}

Catalyst deactivation also produces large changes in its morphology. The deactivation of $\mathrm{Cu}$ catalyst is mainly a result of coke formation, which leads to blocking parts of the active surface. This process predominantly affects fine pores where Knudsen diffusion is the dominant mass transfer mechanism. Therefore, both the effective diffusion coefficient $D_{\text {eff }}$ and the tortuosity factor $\tau$ would change because of the deactivation process.

$\mathrm{Cu}$ catalyst used in aniline production continuously deactivates while in stream with an expected lifetime of $3000 \mathrm{~h}$. The process temperature is continuously increased at small increments to maintain a constant catalyst activity. Total deactivation of the $\mathrm{Cu}$ catalyst in this process is considered when the presence of a very small amount of unreacted nitrobenzene is observed in the reactor effluent stream. The catalyst is discharged from the reactor whenever the reactor effluent stream contains $0.05 \%$ nitrobenzene at a temperature not higher than $230{ }^{\circ} \mathrm{C}$. The discharged catalyst would, therefore, be only partially deactivated and no big change in the measured effective diffusion coefficient $D_{\text {eff }}$ and tortuosity factor $\tau$ of this discharged catalyst from those of freshly reduced catalyst is observed, as shown in Table 4.

\subsubsection{Temperature}

Data of Tables 2 and 3 reveal that $\tau$ values of both catalysts in their oxide and reduced states decrease with increasing the temperature. Measured $\tau$ values at $433 \mathrm{~K}$ for both catalysts in their two studied states are two times smaller than that measured at $293 \mathrm{~K}$. This is attributed in part to resulting increase in gas molecular diffusivity with temperature. Other factors influenced by the temperature increase might also play a role in this outcome.

\subsection{Tortuosity as a tool for design of catalyst pellets and quality control of catalyst preparation}

Pore structures of supported catalysts by in large depend on the pore structure of the support. Various factors and criteria are used in selecting a carrier for a given catalyst. Among the important factors are support surface area and ability to stabilize supported metal particles, chemical properties of the surface, mechanical properties, resistance to the 
reaction media particularly in the regeneration process, thermal stability and resistance to sintering, and porosity and pore size distribution. It is also critical, however, to take into account the effect of selected support on resulting catalyst pellet tortuosity and pore structure at the operating conditions in which the catalyst will be used.

In the same token, design or selection of pelletization methods and conditions should be based on desired outcome of pellet properties including catalyst morphology and structure, tortuosity, pore mean diameter, and other important properties. An important criterion in selecting a pelletization method and pelletizing conditions is to produce catalyst pellets with minimum tortuosity and maximum surface area.

A review of the impact of tortuosity on intraparticle diffusion was recently published by Shen and Chen [7]. The authors discussed the relation between tortuosity and porosity of different solid samples. In this review, the authors quoted the theoretical dependence of tortuosity on catalyst porosity given by Beekman for heterogeneous catalyst as follows [37]:

$$
\tau^{2}=\frac{\alpha}{1-(1-\alpha)^{1 / 8}}
$$

where $\alpha$ is porosity of the pellet. This expression, however, predicts much lower values of tortuosity than those observed experimentally. For example, industrial catalysts usually have a pellet porosity above 0.3 , which according to the Beekman equation should have a tortuosity of only 1.6. This value is obviously substantially lower than experimentally reported values. Therefore, it is clear that up to now, theoretical considerations for predicting heterogeneous catalyst tortuosity are not reliable. The general tendency, however, is that catalyst pellets with smaller porosity should have lower pore tortuosity. Nevertheless, the preparation of pellets with small pore tortuosity is one of the objectives in selecting pelletizing technology and pelletizing conditions.

The pore structure of precipitated catalysts is a result of several consecutive processes like precipitation, aging, filtration, washing, drying, calcination, and pellet shaping. During these processes the catalytically active phases and catalyst pore structure are formed. The needed pore structure of pellets of precipitated catalysts is more difficult to obtain and to reproduce than that of supported catalysts. This is one of the drawbacks of catalyst preparation by co-precipitation by which catalyst reproducibility is difficult to be guaranteed.

To avoid the accidental deviation from standard production in industry it is advisable to include the measurements of the effective diffusion coefficient $D_{\text {eff }}$ and tortuosity factor $\tau$ as a part of compulsory methods for quality control of produced catalysts. The final criterion should be values of these parameters for already activated catalysts at high temperature and high pressure i.e., in conditions close to those at which it is expected to be used. These data can be used as a back feed for correction of preparation procedures.

Recently, using the theoretical considerations, which were experimentally confirmed [38,39], it was shown that for some heterogeneous catalytic processes which proceed on fast deactivating catalysts it is preferable to carry out the reaction in the diffusion controlled conditions because the retardation of deactivation process is higher than that of the main reaction. In this case, the design of such processes strongly depends on knowledge of $D_{\text {eff }}$ and $\tau$, which really represents the mass transfer processes in the reaction conditions. To realize such conditions intentionally the catalyst's pellets should be designed correspondingly to allow proceeding of the reaction into internal diffusion regime.

\section{Conclusions}

The experimental work performed using CM and DMWK for estimation of transport parameters, and the obtained results confirmed that both used methods give compatible results, which render both of these methods as reliable and attractive for industrial laboratory use.

From the above discussion, it follows that the measurements of the effective diffusion coefficients of reagents and tortuosity factor of catalyst pores should be performed taking into account many factors including the following:

- The state of the catalyst sample should correspond to the conditions in which the catalyst is supposed to be used.

- The temperatures, at which the studies are performed, should correspond as much as possible to the catalyst's actual working temperatures.

- Catalyst deactivation by coking and high reaction temperatures leads to a decrease in the tortuosity factor, which implies that catalyst surface area becomes smaller but to some extent with more accessible pores.

If the above factors are not taken into consideration, experimental results may not be very reliable and informative.

It is advisable to use tortuosity factor $\tau$ and effective diffusion coefficients $D_{\text {eff }}$ as one of the criteria for selection of pelletizing technology and for controlling the quality of the produced catalysts.

The effect of different reaction regimes on transport characteristics of the investigated catalysts should obviously be taken into account when kinetics of the reactions is studied, and in reactor modeling and selecting the optimal working conditions of an industrial reactor. 


\section{Symbol description}

A Cross section area of a cylindrical pellet, $\mathrm{cm}^{2}$

$D_{\mathrm{AB}} \quad$ Molecular diffusion coefficient (binary gas mixture), $\mathrm{cm}^{2} / \mathrm{s}$

$D_{\mathrm{A}(r)}$ Composite diffusion coefficient for component A, $\mathrm{cm}^{2} / \mathrm{s}$

$D_{\text {eff }} \quad$ Effective diffusion coefficient, $\mathrm{cm}^{2} / \mathrm{s}$

$D_{\mathrm{KA}(r)}$ Knudsen diffusion coefficient, $\mathrm{cm}^{2} / \mathrm{s}$

$E_{\mathrm{a}} \quad$ Axial dispersion coefficient, $\mathrm{cm}^{2} / \mathrm{s}$

$F \quad$ Space velocity, $\mathrm{cm}^{3} / \mathrm{s}$

$F_{1} \quad$ Space velocity of carrier gas in the upper chamber of the diffusion cell, $\mathrm{cm}^{3} / \mathrm{s}$

$F_{2} \quad$ Space velocity of carrier gas in the lower chamber of the diffusion cell, $\mathrm{cm}^{3} / \mathrm{s}$

$H \quad$ Height of the equivalent theoretical plate (HETP)

$k_{f}^{\text {ext }} \quad$ External mass transfer coefficient, $\mathrm{m} / \mathrm{s}$

$k_{f}^{\text {int }} \quad$ Internal mass transfer coefficient, $\mathrm{m} / \mathrm{s}$

$L \quad$ Pellet length (dynamic method), cm

$M_{\mathrm{a}} \quad$ Molecular weight

$M_{\mathrm{A}} \quad$ Molecular weight of component A

$p \quad$ Pressure

$R \quad$ Gas constant

$r_{\mathrm{e}} \quad$ Equivalent pore radius, $\mathrm{cm}$

$T$ Absolute temperature, $\mathrm{K}$

$t_{0} \quad$ Pulse length, $\mathrm{s}$

$u \quad$ Linear velocity of the carrier gas (chromatographic method), $\mathrm{cm} / \mathrm{s}$

$V_{1} \quad$ Volume of the upper chamber of the diffusion cell, $\mathrm{cm}^{3}$

$V_{2} \quad$ Volume of the lower chamber of the diffusion cell, $\mathrm{cm}^{3}$

$V_{\mathrm{A}} \quad$ Molar volume of component A, $\mathrm{m}^{3} / \mathrm{kmol}$

$V_{\mathrm{B}} \quad$ Molar volume of component $\mathrm{B}, \mathrm{m}^{3} / \mathrm{kmol}$

$\alpha \quad$ Porosity of the catalyst pellet

$\tau \quad$ Tortuosity factor of the catalyst pellet pores

$\mu_{1 \text { corr }}$ First absolute moment, corrected for dead volume, s

$\mu_{\text {1cal }} \quad$ Calculated first absolute moment, $\mathrm{s}$

$\mu_{\text {lobs }}$ Experimentally observed first absolute moment, $\mathrm{s}$

\section{References}

1 Kraushaar-Czarnetzki B, Muller S P. In: de Jong K P Ed. Synthesis of Solid Catalysts. Weinheim: Wiley-VCH, 2009. 173

2 Satterfield C N. Mass Transfer in Heterogeneous Catalysis. Cambridge: MIT Press, 1970
3 Wheeler A. Adv Catal, 1951, 3: 249

4 Carberry J J. In: Anderson J R, Boudart M eds. Catalysis: Science and Technology. Vol. 8. Berlin: Springer, 1987. 131

5 Aris R. Mathematical Theory of Diffusion and Reaction in Permeable Catalysts. I. Theory of Steady State. Oxford: Clarendon Press, 1970

6 Frank-Kamenetzkii D A. Diffusion and Heat Transfer in Chemical Kinetics. 3rd Ed. Moscow: Nauka, 1987

7 Shen L, Chen Z. Chem Eng Sci, 2007, 62: 3748

8 Haynes H W Jr. Catal Rev-Sci Eng, 1988, 30: 563

9 Park I S, Do D D, Rodrigues A E. Catal Rev-Sci Eng, 1996, 38: 189

10 Wijngaarden R J, Kronberg A, Westerterp K R. Industrial Catalysis. Weinheim: Wiley-VCH, 1998

11 Schneider P, Smith J M. AIChE J, 1968, 14: 762

12 Haynes H W Jr, Sharma P N. AIChE J, 1973, 19: 1043

13 Ma Y H, Mancel C. Adv Chem Ser, 1973, 121: 392

14 Hashimoto N, Smith J M. Ind Eng Chem Fundam, 1973, 12: 353

15 Kubin M. Collec Czech Chem Commun, 1965, 30: 1104

16 Kubin M. Collec Czech Chem Commun, 1965, 30: 2900

17 Kucera E. J Chromatogr, 1965, 19: 237

18 Wicke E, Kallenbach R. Kolloid Z, 1941, 97: 135

19 Gubilaro L G, Gioria F, Grego G Jr. Chem Eng J, 1970, 1: 85

20 Dogu G, Smith J M. AEChE J, 1975, 21: 58

21 Dogu G, Smith J M. Chem Eng Sci, 1976, 31: 123

22 Mofat A J. J Catal, 1978, 54: 107

23 Soukup K, Schneider P, Solcova O. Chem Eng Sci, 2008, 63: 1003

24 Hou K, Fowles M, Hughes R. Chem Eng Res Des, 1999, 77: 55

25 Nelder J A, Mead R. Comp J, 1965, 7: 308

26 Petrov L, Kirkov N, Shopov D M. Kinet Katal, 1985, 26: 897

27 Petrov L, Kumbilieva K, Kirkov N. Appl Catal, 1990, 59: 31

28 Wang C T, Smith J M. AIChE J, 1983, 29: 132

29 Hoogschagen J. Ind Eng Chem, 1955, 47: 906

30 Scott D S, Dullien F A L. AIChE J, 1962, 8: 113

31 Diesler P F, Wilhelm R H. Ind Eng Chem, 1953, 45: 1219

32 Metaxas K C, Papayanakos N G. Chem Eng J, 2008, 140: 352

33 Kirszensztejn P, Bell T N. Catal Lett, 1993, 21: 371

34 Twig M V, Spencer M S. Appl Catal A, 2001, 212: 161

35 Inglezakis V J. Int J Chem Reactor Eng, 2010, 8: 1

36 Schimpf S, Muhler M. In: de Jong K P Ed. Synthesis of Solid Catalysts. Weinheim: Wiley-VCH, 2009. 329

37 Beekman J W. Chem Eng Sci, 1990, 45: 2603

38 Kumbilieva K, Kostjukovsky M M, Petrov L, Kiperman S L. Chem Eng Sci, 1988, 43: 1195

39 Kumbilieva K, Sergeeva T Y, Loc L K, Petrov L, Kiperman S L. Appl Catal A, 1992, 82: 159 